\title{
Are small hyperbolic universes observationally detectable?
}

\author{
G.I. Gomero ${ }^{1,2 *}$ M.J. Rebouças ${ }^{1 \dagger}$, R. Tavakol ${ }^{1,3 \ddagger}$ \\ ${ }^{1}$ Centro Brasileiro de Pesquisas Físicas, \\ Rua Dr. Xavier Sigaud 150 \\ 22290-180 Rio de Janeiro - RJ, Brazil \\ ${ }^{2}$ Universidade Católica de Petrópolis, \\ Barão de Amazonas \\ 25685-070, Petrópolis - RJ, Brazil \\ 3 Astronomy Unit, School of Mathematical Sciences, \\ Queen Mary, University of London, \\ Mile End Road, London E1 4NS, UK
}

November 23, 2018

\begin{abstract}
Using recent observational constraints on cosmological density parameters, together with recent mathematical results concerning small volume hyperbolic manifolds, we argue that, by employing pattern repetitions, the topology of nearly flat small hyperbolic universes can be observationally undetectable. This is important in view of the facts that quantum cosmology may favour hyperbolic universes with small volumes, and from the expectation, coming from inflationary scenarios, that $\Omega_{0}$ is likely to be very close to one.
\end{abstract}

It is well known that general relativity is a local metrical theory and therefore the corresponding Einstein field equations do not fix the global topology of spacetime. This freedom has resulted in a great deal of interest in the possibility that the universe may possess compact spatial sections with a non-trivial topology, which do not necessarily have positive curvature (see for example [1] - [3] and references therein).

Interest in such spaces has also come from quantum cosmology, where the existence of the wave function of the universe may require finiteness of the spatial sections (see for

*gomero@cbpf.br, chapita@gft.ucp.br

${ }^{\dagger}$ reboucas@cbpf.br

${ }^{\ddagger}$ r.tavakol@qmw.ac.uk 
example [4]). Also, in the 'tunnelling from nothing' scenario, manifolds with small compact spatial sections may be more likely to emerge [5]. Furthermore, there is a precise sense in which most compact 3-manifolds are hyperbolic [6]. Together these facts have motivated the study of hyperbolic manifolds with non-trivial topology as possible models for our universe.

There has also been two other important developments recently: one observational, the other mathematical. Regarding the former, recent observations seem to indicate that the ratio of the total density to the critical density of the universe, $\Omega_{0}$, is likely to be very close to one [7], with a significant proportion of this energy being in the form of a dark component with negative pressure [8].

Regarding the latter, we first of all discuss briefly some preliminaries and recall that even though at present there is no complete classification of hyperbolic manifolds, a number of important results are known about them, including the two important theorems of Mostow [9] and Thurston [6]. According to the former, geometrical quantities of orientable hyperbolic manifolds, such as their (finite) volumes and the lengths of their closed geodesics, are topological invariants. According to the latter, there is a countable infinity of sequences of compact orientable hyperbolic manifolds, with the manifolds of each sequence being ordered in terms of their volumes [6], with an overall lower bound which is shown to be greater than 0.28151 [10].

A natural way to characterize the shape of such manifolds is in terms of the sizes of their closed geodesics. A particularly useful indicator in this regard is the so called injectivity radius, $r_{i n j}$, defined as the radius of the smallest sphere inscribable in $M$. This in turn allows the definition of a related indicator that has often been utilized in most studies regarding searches for topological multiple images (see, for example [1] - [14]) and references therein), namely the ratio of the injectivity radius to the depth $\chi_{o b s}$ of the astronomical survey up to a given redshift $z_{\max }$

$$
T_{i n j}=\frac{r_{i n j}}{\chi_{o b s}} .
$$

The crucial point regarding this indicator is that, in any universe for which $T_{i n j}>1$, there would be no observed multiple images of either cosmic objects or spots of cosmic microwave background radiation (CMBR), and therefore the topology would not be detectable observationally using pattern repetition, no matter how accurate the observations. Despite the global inhomogeneity of hyperbolic manifolds this result is location independent and therefore applicable to any observer in the universe. Similarly the set of universes for which $T_{i n j}<1$ are observationally detectable through pattern repetitions, at least in principle, for some observers.

An important point regarding $r_{i n j}$ is that its lower bound in the set of all compact hyperbolic manifolds is zero. Thus, no matter what the cosmological parameters (and the resulting $\chi_{o b s}$ ), there will always exist compact hyperbolic universes (with small enough $r_{i n j}$ such that $T_{i n j}<1$ ) such that their topologies are detectable observationally, at least by some observers, even though their number will decrease drastically as $\Omega_{0} \rightarrow 1$ [14.

Now given the potential importance of the small volume hyperbolic manifolds in connection, e.g., with quantum cosmology, the question arises as to whether the existence of detectable topologies for all values of $\Omega_{0}<1$ still holds if we restrict ourselves only to a set of small volume manifolds. Surprisingly, the answer turns out to be in the negative. To see this, we recall an important set of recent mathematical results which show that very small values of $r_{i n j}$ do not occur in small volume hyperbolic manifolds, and that there is in fact a lower bound on the lengths of geodesics in any set of small volume closed orientable hyperbolic 3-manifolds [15, 16]. Thus, for example, according to a theorem of Przeworski [10], the 
shortest geodesic in closed orientable hyperbolic 3-manifolds with volume less than 0.94274 must have length greater than 0.09, corresponding to a lower bound on $r_{i n j}$ of 0.045 . An important point for our purposes here is that it can be shown that there are non-zero lower bounds to lengths of shortest geodesics in any set of closed orientable hyperbolic 3-manifolds, whose volumes are smaller than that of the first orientable cusped manifold by any $\epsilon \neq 0$ [17].

To study an important consequence of these results for cosmology, let us assume that the universe can be modelled by a 4 -manifold $\mathcal{M}$, with a locally hyperbolic isotropic and homogeneous Friedmann-Lemaître-Robertson-Walker metric in the standard form

$$
d s^{2}=-c^{2} d t^{2}+R^{2}(t)\left[d \chi^{2}+\sinh ^{2} \chi\left(d \theta^{2}+\sin ^{2} \theta d \phi^{2}\right)\right] .
$$

Furthermore, let the 3-space be a multiply connected compact quotient manifold of the form $H^{3} / \Gamma$, where $\Gamma$ is a discrete group of isometries of $H^{3}$ acting freely on $H^{3}$. Now in order to have $T_{i n j}$, we consider a survey of depth up to the redshift $z_{\text {max }}$. In this cosmological setting the depth of the survey expressed in units of the curvature radius $\left(\chi_{o b s} \equiv d_{o b s} / R_{0}\right)$ is given by门

$$
\chi_{o b s}=\sqrt{1-\Omega_{0}} \int_{0}^{z_{\max }}\left[(1+x)^{3} \Omega_{m 0}+\Omega_{\Lambda 0}-(1+x)^{2}\left(\Omega_{0}-1\right)\right]^{-1 / 2} d x,
$$

where the current content of the universe is taken to be dust (of density $\rho_{m}$ ) plus a cosmological constant $\Lambda$, with $\Omega_{m}=\frac{8 \pi G \rho_{m}}{3 H^{2}}, \Omega_{\Lambda}=\frac{\Lambda}{3 H^{2}}, \Omega=\Omega_{m}+\Omega_{\Lambda}$, and where the index 0 denotes evaluation at present time.

Now to examine the consequences of the above theorem in the light of recent cosmological observations, recall that as $\Omega_{0} \rightarrow 1$, the curvature radius increases, resulting in a decrease in $\chi_{o b s}$, and as a result the set of topologies that would be observationally detectable would have to possess $r_{i n j}$ approaching zero in order to ensure $T_{i n j}<1$. The above mathematical results, with their lower bounds on $r_{i n j}$, have the consequence that the topology of small hyperbolic universes would be undetectable for values of $\Omega_{0}$ close enough to one. The important point being that the range of $\Omega_{0}$ for which the topology of such universes are undetectable turns out to be within the range of values of $\Omega_{0}$ allowed by recent observations, and particularly those suggested by the inflationary scenarios.

To quantify this, we proceed in the following way. The above theorem gives the lower bound on $r_{i n j}$ in a set of small volume manifolds (those manifolds with volume less than 0.94274, which includes at least the Weeks manifold) to be 0.045 . This allows bounds to be imposed on the ranges of cosmological parameters for which the topology is undetectable. We note that 0.045 is the best estimate available at present and the true lower bound on $r_{i n j}$ may be greater. A way of dealing with this possibility is to consider the first 51 smallest manifolds of the Hodgson-Weeks census of closed hyperbolic manifolds. This set contains all manifolds of the census with volumes smaller than the volume of the first cusped manifold. We note that the volumes of the last 7 manifolds in this set differ from the volume of the first cusped hyperbolic orientable manifold by a factor which is smaller than $10^{-16}$, thus giving an idea of the size of the above mentioned $\epsilon$ for the set of manifolds considered here. The manifold in this set with the lowest $r_{i n j}(=0.152)$ is the eighteenth manifold in the census, denoted by $m 003(-5,4)$, with volume 1.58865 .

Figure 1 gives a plot of the solution curve of equation $\chi_{o b s}=r_{i n j}$ in the $\Omega_{0}-\Omega_{\Lambda 0}$ plane for $r_{i n j}=0.045$ and 0.152 , where a survey of depth $z_{\max }=1200$ (corresponding to the

\footnotetext{
${ }^{1}$ For non-flat models the scale factor $R(t)$ is identified with the curvature radius of the spatial section of the universe at time $t$, and thus $\chi$ can be interpreted as the distance of any point with coordinates $(\chi, \theta, \phi)$ to the origin of coordinates (in $H^{3}$ ), in units of curvature radius, which is a natural unit of length and suitable for measuring areas and volumes. Throughout this letter we shall use this natural unit.
} 
redshift of the surface of last scattering, CMBR) was used. Also included in this Figure is a dashed rectangular box, representing the relevant part (for our purposes here) of the hyperbolic region of the parameter space $\left(\Omega_{0} \in(0.99,1]\right.$ and $\left.\Omega_{\Lambda 0} \in[0.63,0.73]\right)$ given by recent observations [18]. For each value of $r_{i n j}$ undetectablilty is ensured for the values of cosmological parameters (region in the $\Omega_{0}-\Omega_{\Lambda 0}$ plane) which lie above the corresponding curve. Thus for $r_{i n j}=0.045$, all closed orientable hyperbolic manifolds (universes) with volumes less than 0.94274 would have undetectable topology, if the total density $\Omega_{0}$ turned out to be higher than $\sim 0.9998$. On the other hand, for $r_{i n j}=0.152$, the topology of none of the 51 manifolds of the census (whose volumes range from 0.94271 to 2.02988 (corresponding to the manifold $m 010(4,1)$ ), and which includes the Weeks manifold) would be detectable, if the total density $\Omega_{0}$ turned out to be higher than $\sim 0.998$. Clearly, the actual precise bounds on $\Omega_{0}$ depend on the precise value of the $\Omega_{\Lambda 0}$ employed. The resulting allowed changes in the bounds on $\Omega_{0}$ due to employing other values in the observed range of $\Omega_{\Lambda 0}$ are, however, small, as can be seen from Figure 1. Similarly, one can easily find the corresponding ranges of $\Omega_{0}$ for any other particular manifold or finite set of small manifolds. The important point is the existence of a lower bound on $r_{i n j}$ for any finite set of small volume manifolds (smaller, by any $\epsilon \neq 0$, than the volume of the first cusped orientable hyperbolic manifold), which in turn gives lower bounds on $\Omega_{0}<1$, such that the topology of the universe is not detectable with methods based on the search for pattern repetitions.2]

To conclude, if it turns out, as suggested by inflationary scenarios, that $\Omega_{0}$ is very close to one, then our results are significant in implying that the topology of small hyperbolic universes, suggested, for example, by some arguments based on quantum cosmology, can be undetectable using pattern repetitions. This motivates the development of new strategies for looking for the topology of the universe, not based on the observation of repeated patterns.

\section{Acknowledgments}

We are grateful to Andrew Przeworski for very helpful correspondence concerning his work, Jeff Weeks for many useful comments, and Neil Cornish for drawing our attention to the reference [19]. We also thank FAPERJ and CNPq for the grants under which this work was carried out.

\section{Figure caption}

Figure 1 The solutions curve of $\chi_{o b s}=r_{i n j}$, as plots of $\Omega_{0}$ (vertical axis) versus $\Omega_{\Lambda 0}$ (horizontal axis), with $r_{i n j}$ taken as 0.045 (upper curve) and 0.152 (lower curve), respectively. The depth of the survey in both cases correspond to a redshift $z_{\max }=1200$ (CMBR). Included also is a dashed rectangular box, representing the relevant part, for our purposes, of the hyperbolic region of the parameter space given by recent observations. The undetectable region of the parameter space $\left(\Omega_{0}, \Omega_{\Lambda 0}\right)$ corresponding to each value of $r_{i n j}$ lies above the related curve.

\footnotetext{
${ }^{2}$ Note in addition that, following an assertion by Thurston which states that the expectation value for the length of the smallest closed geodesic at an arbitrary point in a generic hyperbolic 3-manifold lies in the range $0.5 \rightarrow 1$, Cornish et al. [19] have argued that even for these large volume hyperbolic manifolds $M$, it is very unlikely that the earth is in a region whose closed geodesics are as short as the shortest closed geodesics of $M$. Thus, according to [14 the chances of detecting the topology of these (large) hyperbolic universes are also very low according to recent observations $\left(\Omega_{0} \sim 1\right)$, even if CMBR is used.
} 


\section{References}

[1] G.F.R. Ellis, Gen. Rel. Grav. 2, 7 (1971); D.D. Sokolov \& V.F. Shvartsman, Sov. Phys. JETP 39, 196 (1974); G.F.R. Ellis \& G. Schreiber, Phys. Lett. A 115, 97 (1986); R. Lehoucq, M. Lachièze-Rey \& J.-P. Luminet, Astron. Astrophys. 313, 339 (1996); B.F. Roukema, Mon. Not. R. Astron. Soc. 283, 1147 (1996); G.F.R. Ellis \& R. Tavakol, Class. Quantum Grav. 11, 675 (1994); N.J. Cornish, D.N. Spergel \& G.D. Starkman, Proc. Nat. Acad. Sci. 95, 82 (1998); N.J. Cornish \& J.R. Weeks, astro-ph/9807311 (1998); G.I. Gomero, A.F.F. Teixeira, M.J. Rebouças \& A. Bernui, gr-qc/9811038 (1998); R. Lehoucq, J.-P. Luminet \& J.-P. Uzan, Astron. Astrophys. 344, 735 (1999); J.P. Uzan, R. Lehoucq \& J.P. Luminet, Astron. Astrophys. 351, 776 (1999); H.V. Fagundes \& E. Gausmann, Phys. Lett. A 261, 235 (1999); M.J.Rebouças Int. J. Mod. Phys. D 9, 561 (2000) G.I. Gomero, M.J. Rebouças \& A.F.F. Teixeira, Phys. Lett. A 275, 355 (2000); G.I. Gomero, M.J. Rebouças \& A.F.F. Teixeira Class. Quantum Grav. 18, 1885 (2001);

[2] R. Lehoucq, J.P. Uzan \& J.P. Luminet, astro-ph/0005515 (2000); V. Blanlœil \& B.F. Roukema, Eds., in the proceedings of the Cosmological Topology in Paris 1998, astroph/0010170. G.D. Starkman, Class. Quantum Grav. 15, 2529 (1998); M. Lachièze-Rey \& J.-P. Luminet, Phys. Rep. 254, 135 (1995);

[3] Ya. B. Zeldovich \& I.D. Novikov, The Structure and Evolution of the Universe, pages 633-640. The University of Chicago Press, Chicago (1983). See also on page 637 of this book the references to earlier works by Süveges (1966), Sokolov (1970), Paal (1971), Sokolov and Shvartsman (1974), and Starobinsky (1975).

[4] D. Atkatz \& H. Pagels, Phys. Rev. D 25, 2065 (1982); Ya.B. Zeldovich \& A.A. Starobinsky, Sov. Astron. Lett. 10, 135 (1984); Y.P. Goncharov \& A.A. Bystsenko, Astrophysics 27, 422 (1989).

[5] G.W. Gibbons, Class. Quantum Grav. 15, 2605 (1998).

[6] W.P. Thurston, Bull. Am. Math. Soc. 6, 357 (1982).

[7] A.E. Lange et al., Phys. Rev. D 63, 042001 (2001); P. de Bernardis et al., in Proceedings of the CAPP2000 conference, Verbier, 17-28 July 2000, astro-ph/0011469 (2000); J.R. Bond et al., in Proceedings of Neutrino 2000 (Elsevier), CITA-2000-63, Eds. J. Law \& J. Simpson, astro-ph/0011381 (2000); J.R. Bond et al., in Proceedings of IAU Symposium 201 (PASP), CITA-2000-65, astro-ph/0011378; A. Balbi et al., Astrophys. J. 545, L1L4 (2000).

[8] B.P. Schmidt et al., Astrophys. J. 50746 (1998); A.G. Riess et al., Astron. J. 116, 1009 (1998); S. Perlmutter et al., Astrophys. J. 517, 565 (1999); S. Perlmutter, M.S. Turner \& M. White, Phys. Rev. Lett. 83, 670 (1999).

[9] G.D. Mostow, Proc. Internat. Congr. Math. Vol 2, 187-197 (1971).

[10] A. Przeworski, Cones Embedded in Hyperbolic 3-Manifolds, preprint available at http://www.ma.utexas.edu/users/prez/.

[11] D.D. Sokolov \& V.F. Shvartsman, Sov. Phys. JETP 39, 196 (1974). 
[12] N.J. Cornish, D.N. Spergel \& G.D. Starkman, Class. Quantum Grav. 15, 2657 (1998).

[13] J.-P. Luminet \& B.F. Roukema, Topology and the Universe: Theory and Observation, astro-ph/9901364.

[14] G.I. Gomero, M.J. Rebouças \& R. Tavakol, Detectability of Cosmic Topology in Almost Flat Universes, gr-qc/0105002. Class. Quantum Grav. 18, in press (2001).

[15] D. Gabai, R. Meyerhoff \& P. Milley, Volumes of Tubes in Hyperbolic 3-Manifolds, preprint available at http://wWw.math.caltech.edu/people/gabaipub.html.

[16] T. Marshall \& G. Martin, Volumes of Hyperbolic 3-folds, preprint (2001).

[17] A. Przeworski, private communication.

[18] J.R. Bond et al., The Quintessential CMB, Past \& Future, in Proceedings of CAPP-2000 (AIP), CITA-2000-64, astro-ph/0011379.

[19] N.J. Cornish, D. Spergel \& G. Starkman, Phys. Rev. D 57, 5982 (1998). 


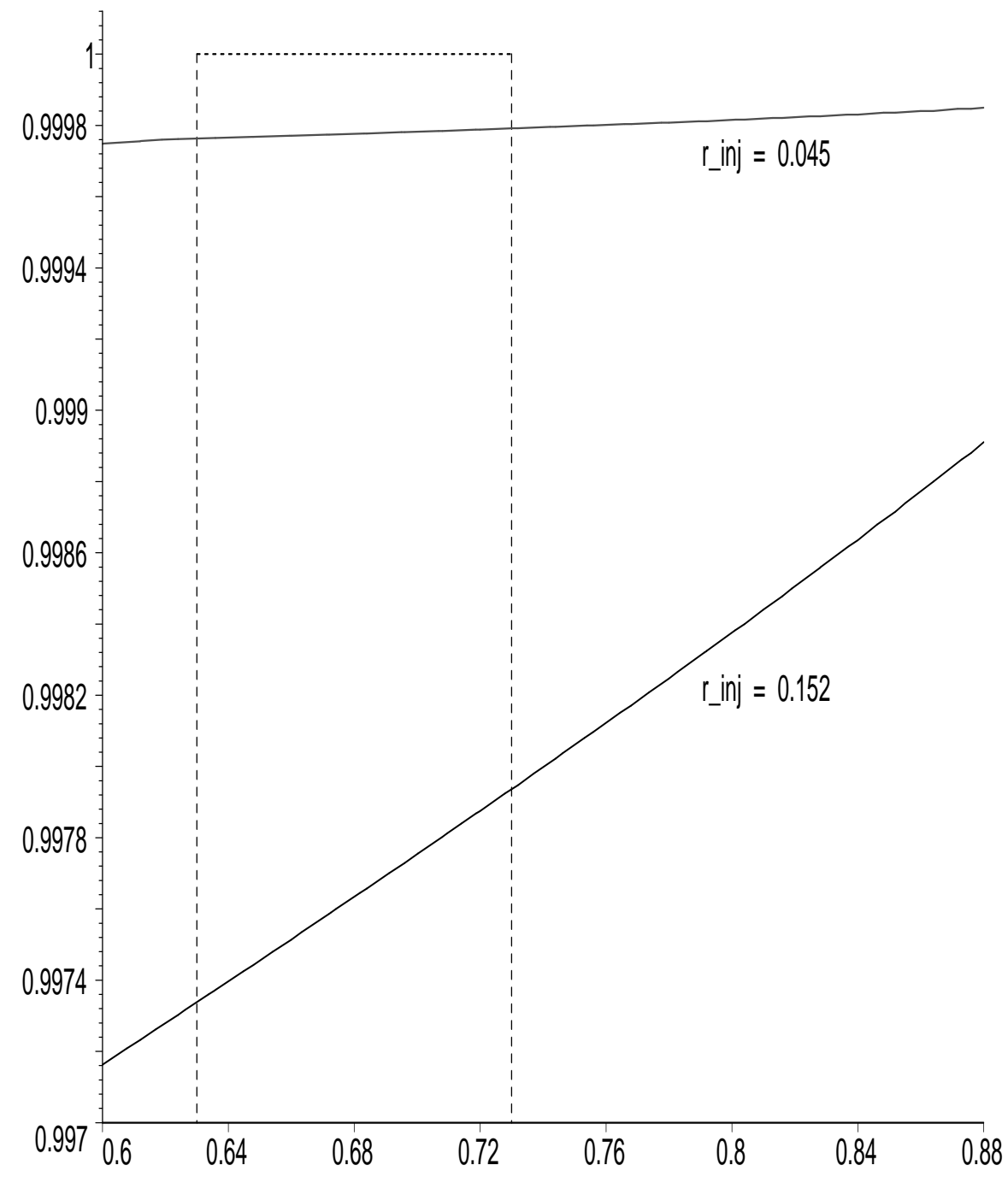

Figure 1 